\title{
Exóticas en humedales: Análisis de las comunidades vegetales de mallines naturales y urbanos en la ciudad de Bariloche
}

\author{
Florencia Cuassolo ${ }^{\bowtie}$ \& Verónica Díaz Villanueva \\ Laboratorio de Limnología, INIBIOMA, Universidad Nacional del Comahue, CONICET, Quintral 1250, Bariloche (8400), \\ Argentina.
}

\begin{abstract}
REsumEn. La vegetación de los humedales desempeña un papel crucial en los ecosistemas andinos, fundamentales por su biodiversidad, por ofrecer numerosos servicios ecosistémicos y por mitigar el cambio climático. A pesar de esto, se conoce muy poco sobre la riqueza y la diversidad de las especies presentes en los humedales y, en particular, en los mallines urbanos. El objetivo de este trabajo fue caracterizar y cuantificar la composición florística de cuatro mallines con distinto grado de urbanización y uso de la tierra (e.g., ganadero, recreativo, turístico, industrial, etc.) dentro del ejido de la ciudad de San Carlos de Bariloche. Se encontró una riqueza total de 81 especies, de las cuales 50\% fueron de origen exótico. Además, nuestros resultados muestran una relación negativa entre la distancia al centro de la ciudad y el porcentaje de especies exóticas. Se señala la presencia de especies nativas exclusivas en cada mallín y se alerta sobre la posible invasión de la especie exótica Potentilla anserina, hallada en los cuatro mallines, lo cual pone en evidencia la necesidad de proteger estos ambientes vulnerables.
\end{abstract}

[Palabras clave: especies invasoras, composición florística, urbanización, impacto antrópico, usos de la tierra]

\begin{abstract}
Exotic species in wetlands: Analysis of plant communities in natural and urban meadows in Bariloche city. Wetland vegetation plays a crucial role in Andean ecosystems due to their biodiversity, to the numerous ecosystemic services they provide and to the climate change mitigation. However, basic knowledge on species richness and diversity in wetland, and in particular in urban meadows, is scarce. The objective of this work was to characterize and quantify floristic composition of four meadows with increasing distance to the city and different land uses (e.g., grazing, recreational, touristic, industrial, etc.) in San Carlos de Bariloche City. We identified 81 species, $50 \%$ of which were exotic. Also, our results showed a negative relation between the distance to the city center and the percentage of exotic species. We pointed to the presence of native species exclusive of each meadow, and alerted to the possible invasion of the exotic species Potentilla anserina, found in the four meadows, which highlighs the necessity of protection of these vulnerable environments.
\end{abstract}

[Palabras clave: invasive species, floristic composition, urbanization, anthropic impacts, land uses]

\section{INTRODUCCIÓN}

Las invasiones biológicas, entendidas como la llegada, colonización y expansión de especies más allá de sus rangos biogeográficos naturales, son consideradas uno de los principales componentes del cambio global (Sala 2000). Un fenómeno de este cambio es el transporte masivo de personas y de mercaderías, las que, además de las aves y los animales, actúan como vectores de dispersión. Una vez establecidas, estas especies causan impactos notables sobre las comunidades nativas y la sociedad humana (Simberloff et al. 2013). Existe abundante evidencia empírica que indica que las especies invasoras pueden afectar la biodiversidad (Sala 2000; Lockwood et al. 2009), causar extinciones de especies, daños en poblaciones de organismos silvestres o domesticados y alterar los ecosistemas.
Los humedales son sitios de alta biodiversidad vegetal y animal (Ramsar 2006). La convención RAMSAR, un tratado intergubernamental que sirve de marco para la acción nacional y la cooperación internacional en pro de la conservación y el uso racional de los humedales y sus recursos, señala que los humedales sirven de sumidero al $40 \%$ del carbono que se genera en el planeta. Si se los destruye dejarían de eliminarse cantidades importantes de gases de efecto invernadero y, por ende, aumentaría el potencial de retención del calor en la atmósfera, con implicancias en el calentamiento global y el aumento de la temperatura. Además, debido tanto a los disturbios naturales (fluctuaciones hídricas) como a los antrópicos (ganado, urbanización), son ambientes muy propensos a ser invadidos por especies exóticas (Raffaele 2004; Zedler and Kercher 2004; Gallardo et al. 2016), las cuales 
pueden alterar la composición y estructura de las comunidades de los humedales (Vitousek 1990). La susceptibilidad de los humedales a ser invadidos por especies exóticas se refleja en el hecho de que un alto porcentaje (24\%) de las plantas más invasoras del mundo son invasoras de humedales; forman monotipos que alteran por completo la estructura del ambiente, disminuyen la biodiversidad (tanto en número como en "calidad"), cambian el ciclo de los nutrientes y la productividad (a menudo la aumentan) y modifican las redes alimentarias (Zedler and Kercher 2004).

Otro componente del cambio global es el incremento exponencial en la población, acompañado por cambios en el uso de la tierra y en la cobertura vegetal; esto impacta sobre los ecosistemas naturales en múltiples escalas espaciales (Faulkner 2004). En las últimas décadas, la población humana aumentó hasta un 50\% (Ehrenfeld 2000), sobre todo en las áreas costeras. Las actividades humanas como la urbanización, la agricultura, la ganadería, las industrias, etc. descargan contaminantes en forma disuelta y materia en suspensión a los cuerpos de agua, lo que disminuye la calidad del agua (Harguinteguy et al. 2013). Los humedales son ambientes muy vulnerables a las diversas actividades antrópicas (antropización); en este sentido, la urbanización es la principal causa de pérdida de estos ambientes debido a que para poder ser utilizados como terrenos edificables se los drena, rellena y deseca, se destruye su vegetación y se contaminan sus aguas y sedimentos (Ramírez et al. 2002). En el último siglo desapareció más de la mitad de la superficie de humedales en todo el mundo; actualmente quedan entre 600 y 900 millones de hectáreas, de las cuales 60 a 70 millones (menos del 10\%) se encuentran protegidas (Ramsar 2006).

Un tipo de humedal característico de la Región Patagónica es el mallín (término mapuche que significa bañado o pantano), cuyo nivel de agua es muy fluctuante, ya que puede pasar un período del año sin agua en superficie. Los mallines juegan un papel clave en los ecosistemas por su alta biodiversidad (Perotti et al. 2005; Jara et al. 2013) y su producción primaria neta elevada (Raffaele 1999; Buono et al. 2010). Se encuentran ubicados en toda la Patagonia, en un rango amplio de precipitaciones que va desde los $2000 \mathrm{~mm}$ / año en la cordillera andina al oeste, hasta la estepa (al este), con menos de $200 \mathrm{~mm}$ de precipitación anual (Paruelo et al. 1998). Se caracterizan por la presencia de especies vegetales adaptadas a las fluctuaciones del nivel del agua (hidrófitas) y la ausencia de aquellas no tolerantes a la condición de anegamiento producto de los pulsos de inundación. Suelen presentar una cobertura vegetal densa, dominada principalmente por plantas herbáceas de las familias Juncaceae, Cyperaceae y Poaceae (Iriondo et al. 1974; Raffaele 1999).

En particular, la ciudad de San Carlos de Bariloche posee numerosos mallines de bosque con diferente grado de disturbio antrópico. Pese a que la Carta Orgánica Municipal estipula en su Artículo 185 que "la Municipalidad protege y regula el uso racional de los humedales dentro del ejido, favoreciendo el mantenimiento de los mismos", en la práctica, las técnicas de manejo y regulación son casi inexistentes. Al presente existen sólo dos informes técnicos realizados por la Universidad Nacional del Comahue que caracterizan la situación de dos mallines de la ciudad: el Mallín de Bahía Serena (González and Alonso 2009) y el Complejo Morenito-Trébol- Ezquerra (Balseiro 2014), y no se realizaron estudios de la flora de estos ambientes ni se realizó un análisis de los diferentes impactos ambientales de origen antrópico a los que están sometidos. En las últimas décadas, el área urbana de la ciudad de Bariloche creció (Guevara et al. 2005) y sustituyó sistemáticamente distintas superficies de bosque nativo, incluyendo entre ellas a los mallines. Nuestro principal objetivo fue relevar y comparar la flora presente en cuatro mallines dentro del ejido municipal de San Carlos de Bariloche con distinto grado de impacto antrópico, y determinar si existe una relación entre el grado de antropización (estimada como distancia al centro de la ciudad) y la cantidad de especies de plantas exóticas. Nuestra hipótesis principal es que el impacto antrópico afecta la riqueza y la abundancia de las especies, en detrimento de las especies nativas.

\section{Materiales y MÉTOdos}

\section{Área de estudio}

El estudio se llevó a cabo en la ciudad de San Carlos de Bariloche, ubicada dentro del Parque Nacional Nahuel Huapi, en el noroeste de la Patagonia argentina $\left(40^{\circ} 20^{\prime} \mathrm{S}\right.$ - $41^{\circ} 35^{\prime} \mathrm{S}$ y $\left.71^{\circ} 02^{\prime} \mathrm{O}-71^{\circ} 56^{\prime} \mathrm{O}\right)$. El clima de la 
región es templado frío, con una precipitación media anual de $1500 \mathrm{~mm}$ (Paruelo et al. 1998), concentrada en otoño en forma de lluvias y en invierno como lluvias y nevadas, y veranos secos. El tipo de vegetación a lo largo del ejido municipal va desde bosque húmedo a bosque de transición. En el bosque húmedo predominan las especies perennifolias generalmente dominado por Nothofagus dombeyi (coihue) que ocupa las orillas de los lagos y las zonas bajas de las laderas hasta los $1000 \mathrm{~m} \mathrm{~s}$. n. m., y arbustos como Luma apiculata (arrayán), Fuchsia magellanica (chilco) y Chusquea culeou (caña coligüe). En el bosque de transición predominan las especies de hoja caduca como Nothofagus antarctica (ñire) y Embothrium coccineum (notro), y especies perennifolias como Maytenus boaria (maitén), Lomatia hirsuta (radal) y la conífera
Austrocedrus chilensis (ciprés de la cordillera) (Ezcurra and Brion 2005). Los suelos son de origen volcánicos (andisoles) con un bajo grado de desarrollo y con alta capacidad para estabilizar la materia orgánica, almacenar agua, amortiguar el $\mathrm{pH}$ y retener el fósforo (Mazzarino et al. 1998; Satti et al. 2003).

Se seleccionaron cuatro mallines con distinto grado de urbanización, ubicados dentro del ejido municipal de San Carlos de Bariloche (Figura 1). El hidroperíodo de estos mallines (tiempo en el que el mallín presenta agua superficial) depende principalmente de las precipitaciones, y varía entre 9 a 12 meses; el momento de mayor sequía es el verano. En su fase acuática, los mallines presentan características similares en cuanto a temperatura, $\mathrm{pH}$ y conductividad (Jara et al. 2013).

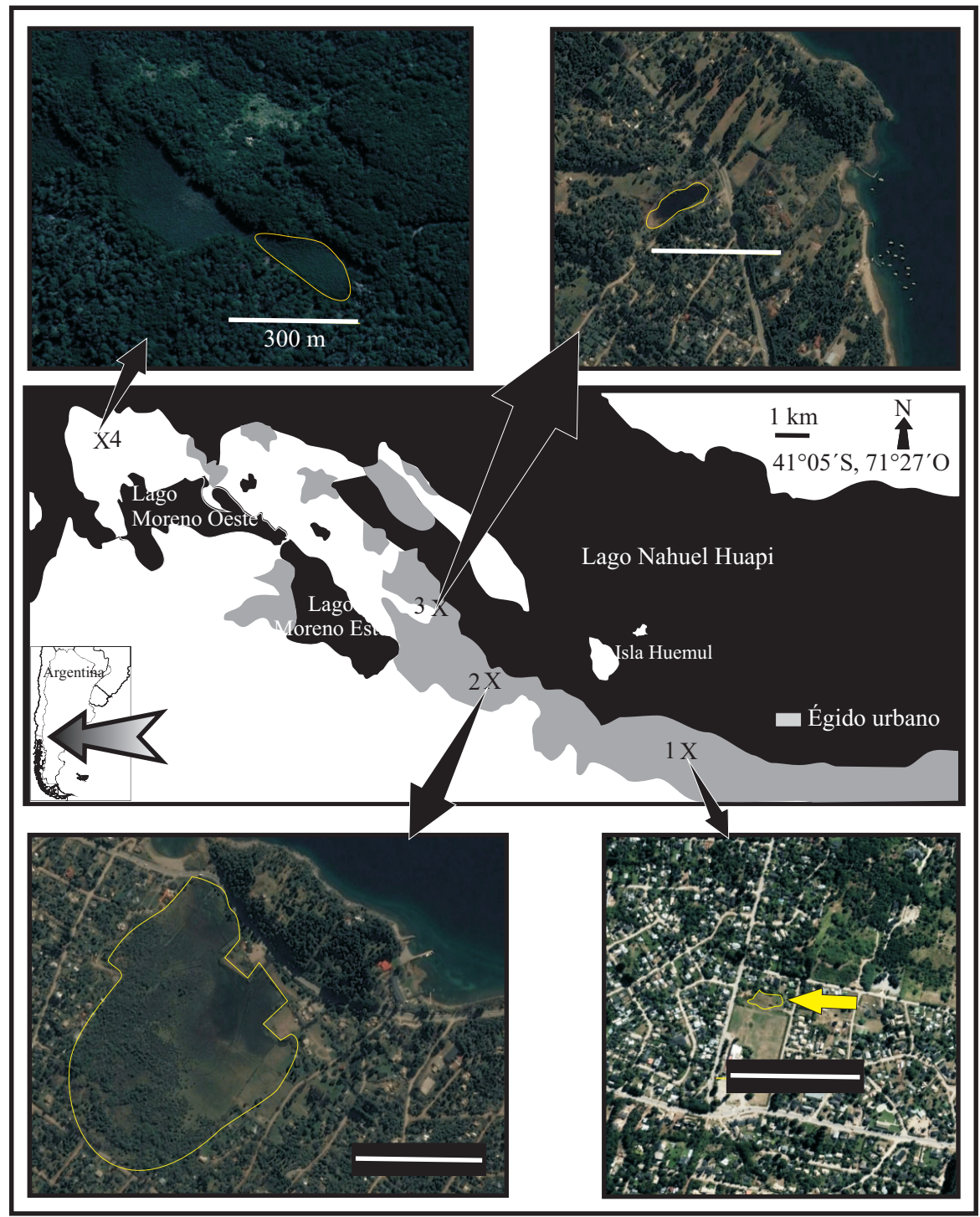

Figura 1. Sitios de estudio dentro del ejido municipal de la ciudad de Bariloche. 1: Teleférico, 2: Bahía Serena, 3: Fantasma, 4: Llao-Llao. Las áreas grises indican urbanización.

Figure 1. Study sites within the San Carlos de Bariloche City. Teleférico, 2: Bahía Serena, 3: Fantasma, 4: Llao-Llao. Grey areas indicate urbanization. 
Tabla 1. Características y usos de los mallines estudiados. Referencias: U (urbanización), R (recreativo); G (ganado); $\mathrm{T}$ (turístico).

Table 1. Characteristics of the wetlands studied. References: U (urbanization), R (recreative); G (cattle); $\mathrm{T}$ (tourist).

\begin{tabular}{lcccc}
\hline & Teleférico & Serena & Fantasma & Llao-Llao \\
\hline Área (ha) & 0.17 & 21 & 1 & 2.2 \\
Número de casas & 30 & 1200 & 60 & 0 \\
$\begin{array}{l}\text { Densidad casas } \\
\text { (nro. de casas/ha) }\end{array}$ & 176 & 60 & 5 & 0 \\
Usos & $\mathrm{U}, \mathrm{R}, \mathrm{G}, \mathrm{T}$ & $\mathrm{U}, \mathrm{G}, \mathrm{T}$ & $\mathrm{R}, \mathrm{U}$ & Ninguno \\
\hline
\end{tabular}

El Mallín Teleférico $\left(41^{\circ} 07^{\prime} \mathrm{S}-7^{\circ} 22^{\prime} \mathrm{O}, 827\right.$ m s. n. m.) (Figura 1.1) es semipermanente; se localiza en la ladera norte del Cerro Otto, a $5 \mathrm{~km}$ del centro de la ciudad. Este mallín presenta un ojo de agua permanente (área $=$ $380 \mathrm{~m}^{2}$, profundidad máxima $=1 \mathrm{~m}$ ) rodeado de una gran zona de drenaje en forma de pradera o pastizal donde se implanta un importante centro turístico, la estación de cablecarril del Teleférico; esto determina un uso intensivo de la tierra (Tabla 1). Se observa la presencia de ganado equino en el verano (entre 2 y 5 individuos).

Por su parte, el Mallín Bahía Serena (4106오 - 77²6’ O, 771 m s. n. m.) (Figura 1) también es semipermanente; se ubica a $12 \mathrm{~km}$ del centro de la ciudad. Posee una zona de anegamiento permanente (área $=15 \mathrm{~m}^{2}$, profundidad máxima $=1.5 \mathrm{~m}$ ) que recibe vuelcos de las casas ubicadas al oeste, mientras que el resto de la superficie de este humedal presenta un profundo grado de modificación. La construcción de la Avenida Bustillo alteró la conexión que este mallín tenía con el Lago Nahuel Huapi, lo que definió su aspecto actual. Además, sufrió eventos de rellenado en el sector norte para la urbanización de los lotes. También los emprendimientos turísticos y gastronómicos que se emplazan en las proximidades y sobre el humedal implican mayores volcados de materia orgánica, con consecuencias serias para la calidad ambiental en general (González and Alonso 2009). Hay presencia de ganado equino durante el verano (entre 20 y 30 individuos).

La Laguna Fantasma $\left(41^{\circ} 05^{\prime} \mathrm{S}-71^{\circ} 26^{\prime}\right.$ O, 779 m s. n. m.) (Figura 1) es un mallín temporario localizado a $15 \mathrm{~km}$ del centro de la ciudad. Alcanza una profundidad máxima de $2 \mathrm{~m}$, mientras que en el verano permanece completamente seco y se asemeja a un ambiente terrestre. En 1994 fue declarado Reserva Intangible Municipal (Ordenanza $N^{\circ}$
332-CM-94). La presencia de ganado equino es muy esporádica.

Por último, el Mallín de Llao-Llao (410` S $71^{\circ} 33^{\prime} \mathrm{O}, 764 \mathrm{~m}$ s.n. m.) (Figura 1) es un mallín temporario emplazado en un claro de bosque a $25 \mathrm{~km}$ del centro de la ciudad, sin ningún tipo de urbanización. Este mallín se halla inserto en el Parque Municipal Llao-Llao. Alcanza su profundidad máxima de $0.4 \mathrm{~m}$ en los meses de invierno y, al igual que Laguna Fantasma, se seca completamente en los meses de verano. La ausencia de ganado es total.

\section{Muestreo}

La superficie de los mallines fue calculada por medio de imágenes satelitales (Google Earth) y se corroboraron las medidas de largo y ancho máximo a campo utilizando cinta métrica. Mediantelas mismasimágenes y observaciones a campo se identificaron tres zonas del humedal: central (o inundada), intermedia y periférica. Los mallines se muestrearon al final de la estación de crecimiento (finales del verano). En una primera instancia se realizó un muestreo cualitativo, para lo cual se recorrieron los ambientes en su totalidad y se colectó material vegetal para identificar las especies y realizar un herbario; este herbario se encuentra en el laboratorio de Limnología de la Universidad Nacional del Comahue. Además, se clasificaron las especies según su origen biogeográfico en nativas y exóticas utilizando bibliografía acerca del origen y la distribución de las especies. La identificación de las plantas recolectadas consistió en observaciones generales de las estructuras exomorfológicas y anatómicas de los órganos vegetales, con especial interés en los caracteres de posible valor diagnóstico (Correa 1971-1998; Dimitri 1977; Boelcke and Vizinis 1986).

Luego de las identificaciones se realizó un muestreo cuantitativo en el que se estimó la cobertura utilizando un cuadrado de 50×50 $\mathrm{cm}\left(0.25 \mathrm{~m}^{2}\right)$ cada 5 metros a lo largo de 3 transectas longitudinales y 5-10 transversales (Matteucci and Colma 1982) que incluían las zonas centrales e intermedias de los ambientes. La zona periférica no se incluyó en el muestreo de cobertura, pero sí se relevó la riqueza vegetal de este sector. En total se relevaron entre 20 y 40 cuadrados para las transectas longitudinales, y 20 para las transversales. Cuando la vegetación no se pudo identificar, se cuantifico un porcentaje de "spp. no identificadas". De igual manera, se cuantifico el porcentaje de suelo desnudo. 
Dentro de la variable antropización se consideraron los usos del suelo como la urbanización (número de casas), ganado (número de caballos), recreativo (parquizaciones) y turístico (cabañas de alquiler turístico, restaurantes, teleférico, etc.). La urbanización se calculó contabilizando el número de casas a una distancia de 100 $\mathrm{m}$ desde la zona periférica de cada mallín mediante imágenes satelitales (Google Earth) y observación directa. Para estimar la densidad poblacional se contabilizó el número de casas por hectárea rodeando cada humedal. También se tomó la distancia al centro de la ciudad como una variable indicadora de grado de antropización.

\section{Análisis de datos}

La riqueza específica fue considerada como el número total de especies en cada sitio. Se calculó la diversidad utilizando los datos de cobertura de cada especie como una medida de abundancia y aplicando el índice de Shannon. Se cuantificó la riqueza total de especies de cada ambiente y se estimó la proporción de nativas y exóticas. Se analizó la riqueza de especies en relación con el tamaño del mallín y con la distancia al centro de la ciudad, y el porcentaje de especies exóticas en relación con la distancia de cada sitio al centro de la ciudad mediante regresión lineal. Para evaluar cuáles especies de plantas de los mallines contribuyeron a las diferencias entre ellos, se realizó un análisis del gradiente (análisis de componentes principales, PCA) utilizando los porcentajes de cobertura de todas las especies, mediante el paquete estadístico PRIMER 6.

\section{Resultados}

Los cuatro mallines estudiados difirieron tanto en sus dimensiones como en el grado de antropización (Tabla 1, Figura 1). Si bien el mayor número de casas se contabilizaron en el mallín Bahía Serena, la densidad de casas fue superior en el Teleférico, que es el más cercano al centro de la ciudad. Otros aspectos relacionados con la antropización, además de la urbanización, fueron la presencia de ganado, mayor en el de Bahía Serena y nulo en el del Llao-Llao, y el uso turístico y recreativo, que impactan tanto al mallín de Bahía Serena como al Teleférico, y en menor medida a la Laguna Fantasma.

Se determinó un total de 83 especies pertenecientes a 33 familias. De este total, el
$50 \%$ de las especies fueron de origen exótico (Tabla 2). La familia con mayor número de especies fue Poaceae (14), seguida por Cyperacea (7), Asteracea y Rosaceae (6 especies cada una). El mayor número de especies se encontró en el mallín Bahía Serena, mientras que el mallín Teleférico y el Llao-Llao tuvieron la menor cantidad de especies (Tabla 2). El mayor número de especies exóticas se halló en el mallín Teleférico (Figura 2). Por otro lado, la menor diversidad se halló en Laguna Fantasma (Tabla 2). La riqueza de especies en cada sitio se relaciónó positivamente con el tamaño del mallín $\left(\mathrm{R}^{2}=0.86, P=0.010\right)$, pero no con la densidad de casas $\left(R^{2}=0.13 ; P>0.05\right)$ (Figura 3). Sin embargo, se observó una fuerte relación negativa entre el porcentaje de especies exóticas y la distancia al centro de la ciudad $\left(\mathrm{R}^{2}=0.98 ; P=0.009\right)$ (Figura 3$)$.

El mallín Teleférico es el más pequeño (Tabla 1, Figura 1) y, al mismo tiempo, es el más cercano al centro de la ciudad. Presentó una riqueza total de 24 especies, de las cuales el $70 \%$ fue de origen exótico; este valor supera al resto de los ambientes. Además, del total

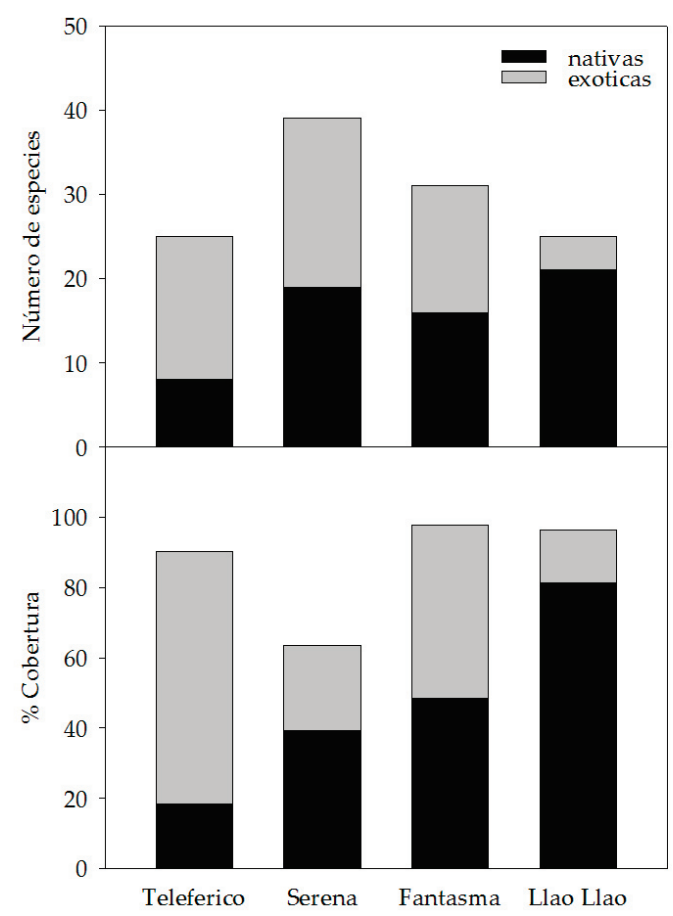

Figura 2. Número de especies y porcentaje de cobertura de nativas y exóticas en los mallines estudiados. El porcentaje de cobertura de las nativas y exóticas no suman $100 \%$ porque se consideró al suelo desnudo dentro del total de cobertura del mallín.

Figure 2. Number of native and exotic species in the studied wetlands.The percentage of native and exotic coverage do not add up to $100 \%$ because the bare soil is considered within the total coverage of the wetland. 
Tabla 2. Porcentaje de cobertura de las especies presentes en cuatro mallines urbanos del ejido municipal de la ciudad de San Carlos de Bariloche. Origen: N: Nativas, E: Exóticas. (*): exclusiva de un solo ambiente. X: presencia de las especies presentes en zona perisférica (\#): especies con más de un 10\% de cobertura.

Table 2. Floristic list present in four urban wetlands of the San Carlos de Bariloche city. Origin: N: Native, E: Exotic. $(*)$ : exclusive of a single environment. X: presence of the species in the intermediate zone. (\#): species with more than $10 \%$ of coverage.

\begin{tabular}{|c|c|c|c|c|c|c|}
\hline Familia & Especie & Origen & Teleférico & Serena & Fantasma & Llao-Llao \\
\hline Apiaceae & Hydrocotile chamaemorus & $\mathrm{N}$ & & 0.23 & & 7.52 \\
\hline \multirow{6}{*}{ Asteraceae } & $\begin{array}{l}\text { Isolepis ranko" } \\
\text { Chrisantemum leucantemum }\end{array}$ & $\begin{array}{l}N \\
E\end{array}$ & & 0.96 & & \\
\hline & Hypochaeris radicata & $\mathrm{E}$ & & 0.02 & & \\
\hline & Matricaria inodora & E & 1.36 & & & \\
\hline & Symphiotricum vahlii & $\mathrm{N}$ & & 0.02 & & 0.34 \\
\hline & Tanacetum vulgare & $\mathrm{E}$ & $\mathrm{x}$ & & & \\
\hline & Taraxacum officinalis & E & 0.52 & 0.25 & $\mathrm{x}$ & \\
\hline Berberidaceae & Berberis microphylla & $\mathrm{N}$ & 0.43 & 1.75 & & \\
\hline \multirow[t]{2}{*}{ Betulaceae } & Alnus glutinosa & $\mathrm{E}$ & & $\mathrm{x}$ & & \\
\hline & Betula pendula & E & & $\mathrm{x}$ & & \\
\hline \multirow[t]{2}{*}{ Brasicacea } & Cardamine tenuirostris* & $\mathrm{N}$ & & 0.02 & & \\
\hline & Nasturtium officinale & $\mathrm{E}$ & & 0.02 & & \\
\hline Cariophyllaceae & Sagina procumbens & E & & 2.45 & & \\
\hline Celastraceae & Maytenus boaria & $\mathrm{N}$ & $\mathrm{x}$ & $\mathrm{x}$ & $\mathrm{x}$ & \\
\hline Chenopodiaceae & Chenopodium album & $\mathrm{E}$ & 0.43 & & & \\
\hline Combolbulaceae & Calistegia sepium & E & 0.43 & & & \\
\hline \multirow[t]{2}{*}{ Cupressaceae } & Austrocedrus chilensis & $\mathrm{N}$ & & & $\mathrm{x}$ & \\
\hline & Juniperus comunis & E & & $\begin{array}{c}x \\
089\end{array}$ & & \\
\hline \multirow{4}{*}{ Cyperaceae } & Carex barrosi & $\mathrm{N}$ & 0.02 & 0.02 & $20.12 \#$ & $\begin{array}{c}23.1 / 7 \text { \# } \\
0.02\end{array}$ \\
\hline & Eleocharis macrostachya & $\mathrm{N}$ & & & 0.07 & 0.02 \\
\hline & Eleocharis pachycarpa & $\mathrm{N}$ & & 4.18 & 18.76\# & 1.70 \\
\hline & Schoenoplectus californicus & $\mathrm{N}$ & $17 \#$ & 0.02 & 0.44 & 2.41 \\
\hline Escalloniaceae & Escallonia virgata & $\mathrm{N}$ & $\mathrm{x}$ & $\mathrm{x}$ & & $\mathrm{x}$ \\
\hline \multirow[t]{4}{*}{ Fabaceae } & Lotus uliginosus & $\mathrm{E}$ & & & & 2.27 \\
\hline & Retama sphaerocarpa & E & & $\mathrm{x}$ & & \\
\hline & Trifolium pratense & $\mathrm{E}$ & 7.26 & 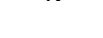 & & \\
\hline & Trifolium repens & E & 0.02 & 5.07 & & \\
\hline Fagaceae & Quercus sp. & E & & & $\mathrm{x}$ & \\
\hline Funariaceae & musgo Funaria sp. & ? & & 1.30 & & \\
\hline \multirow[t]{4}{*}{ Juncaceae } & Juncus procerus & $\mathrm{N}$ & & 0.02 & 0.44 & 2.73 \\
\hline & Juncus balticus* & $\mathrm{N}$ & & 0.02 & & \\
\hline & Juncus bufonius* & $\mathrm{N}$ & & 24.70\# & & \\
\hline & Juncus dombeyanus* & $\mathrm{N}$ & & & & $16.86 \#$ \\
\hline \multirow[t]{3}{*}{ Lamiaceae } & Menta x piperita & $\mathrm{E}$ & 0.43 & & & \\
\hline & Mentha spicata & E & & 0.26 & & \\
\hline & Prunnela vulgaris & E & 0.43 & & & \\
\hline \multirow[t]{2}{*}{ Myrtaceae } & Luma apiculata & $\mathrm{N}$ & & & & $\mathrm{x}$ \\
\hline & Myrceugenia exsucca & $\mathrm{N}$ & & & & $\mathrm{x}$ \\
\hline \multirow[t]{2}{*}{ Nothofagaceae } & Nothofagus antarctica & $\mathrm{N}$ & & $\mathrm{x}$ & & $\mathrm{x}$ \\
\hline & Nothofagus dombeyi & $\mathrm{N}$ & & $\mathrm{x}$ & $\mathrm{x}$ & $\mathrm{x}$ \\
\hline Oleaceae & Fraxinus americana & $\mathrm{E}$ & & & $\mathrm{x}$ & \\
\hline Onagraceae & Epilobium australe var. puberulum & $\mathrm{N}$ & & 0.37 & & 0.02 \\
\hline Pinaceae & Pinus sp. & $\mathrm{E}$ & & $\mathrm{x}$ & $\mathrm{x}$ & \\
\hline \multirow[t]{3}{*}{ Plantaginaceae } & Plantago australis subsp. cummingiana & $\mathrm{N}$ & & 6.05 & & \\
\hline & Plantago australis var. cumingiana & $\mathrm{N}$ & & & & 0.68 \\
\hline & Plantago lanceolata & $\mathrm{E}$ & 0.43 & & & \\
\hline \multirow[t]{14}{*}{ Poaceae } & Agropyron repens & $\mathrm{E}$ & & & 0.01 & \\
\hline & Agrostis afin vidali* & $\mathrm{N}$ & & 0.96 & & \\
\hline & Agrostis exasperata & $\mathrm{N}$ & 0.43 & & 0.15 & 0.02 \\
\hline & Agrostis stolonifera & $\mathrm{E}$ & & & 0.15 & 9.77 \\
\hline & Alopecurus magellanicus* & $\mathrm{N}$ & & & 0.01 & \\
\hline & Alopecurus pratensis & $\mathrm{E}$ & & & & 2.27 \\
\hline & Festuca purpurasens* & $\mathrm{N}$ & & & & 0.23 \\
\hline & Holcus lanatus & $\mathrm{E}$ & & 2.46 & & \\
\hline & Hordeum sp. & $?$ & & & 0.96 & \\
\hline & Lolium perenne & $\mathrm{E}$ & & & 1.93 & \\
\hline & Роа аппиа & $\mathrm{E}$ & 37.82 & & & \\
\hline & Polypogon australis* & $\mathrm{N}$ & & & & 3.48 \\
\hline & Polypogon monspeliencis & $\mathrm{E}$ & & 5 & & \\
\hline & Puccinellia glaucesens* & $\mathrm{N}$ & & & 2.67 & \\
\hline Poligonaceae & Poligonum aviculare & $\mathrm{E}$ & 0.43 & & & \\
\hline & Rumex crispus & E & 0.43 & 0.02 & & \\
\hline Proteaceae & Lomatia hirsuta & $\mathrm{N}$ & & & $\mathrm{x}$ & \\
\hline Ranunculaceae & Ranunculus repens & $\mathrm{E}$ & 18.30\# & & 0.36 & \\
\hline & Ranunculus trichophyllum* & $\mathrm{N}$ & & & 0.20 & \\
\hline
\end{tabular}


Tabla 2. Continuación.

Table 2. Continuation.

\begin{tabular}{|c|c|c|c|c|c|c|}
\hline Familia & Especie & Origen & Teleférico & Serena & Fantasma & Llao-Llao \\
\hline Rhamnaceae & Discaria chacaye & $\mathrm{N}$ & $\mathrm{x}$ & & & \\
\hline \multirow[t]{6}{*}{ Rosaceae } & Crataegus monogyna & $\mathrm{E}$ & & & $\mathrm{x}$ & \\
\hline & Fragaria chiloensis & $\mathrm{N}$ & & 0.18 & & \\
\hline & Malus domestica & $\mathrm{N}$ & & & $\mathrm{x}$ & \\
\hline & Potentilla anserina & $\mathrm{E}$ & 3.48 & 8.12 & 46.65\# & 0.68 \\
\hline & Rosa rubiginosa & E & $\mathrm{x}$ & $\mathrm{x}$ & $\mathrm{x}$ & \\
\hline & Sorbus sp. & E & & & $\mathrm{x}$ & \\
\hline \multirow[t]{3}{*}{ Rubiaceae } & Gallium aparine & E & & 0.11 & & \\
\hline & Leptostigma arnottianum* & $\mathrm{N}$ & & & 0.66 & \\
\hline & Gálium magellanicum & $\mathrm{N}$ & & & 0.01 & 4.91 \\
\hline Salicaceae & Populus alba & E & & & $\mathrm{x}$ & \\
\hline \multirow{3}{*}{$\begin{array}{l}\text { Sapindaceae } \\
\text { Scrofulariaceae }\end{array}$} & Salix fragilis & E & $\mathrm{x}$ & $\mathrm{x}$ & $\mathrm{x}$ & \\
\hline & Acer pseudoplatanus & E & & & $\mathrm{x}$ & \\
\hline & Veronica anagallis-aquatica & E & 0.43 & 0.37 & & \\
\hline \multirow{4}{*}{33 familias } & $\%$ cobertura spp. sin determinar & & 13 & 29 & 2 & 0 \\
\hline & $\%$ suelo desnudo & & 3 & 1 & 0 & 5 \\
\hline & Diversidad & & 2.04 & 2.44 & 1.39 & 2.38 \\
\hline & Total 81 especies & & 25 & 40 & 32 & 25 \\
\hline
\end{tabular}

de la cobertura, un alto porcentaje $(68 \%)$ correspondió a las exóticas (Tabla 2, Figura 2). Uno de los impactos observados en este mallín fue el pastoreo por ganado equino, lo que dificultó las tareas de identificación ( $20 \%$ sin identificar). El ojo de agua permanente se encuentra colonizado principalmente por Schoenoplectus californicus (17\% de cobertura). En la zona litoral o intermedia de la laguna se encontró una dominancia de especies exóticas como Poa annua (37\%), Ranunculus

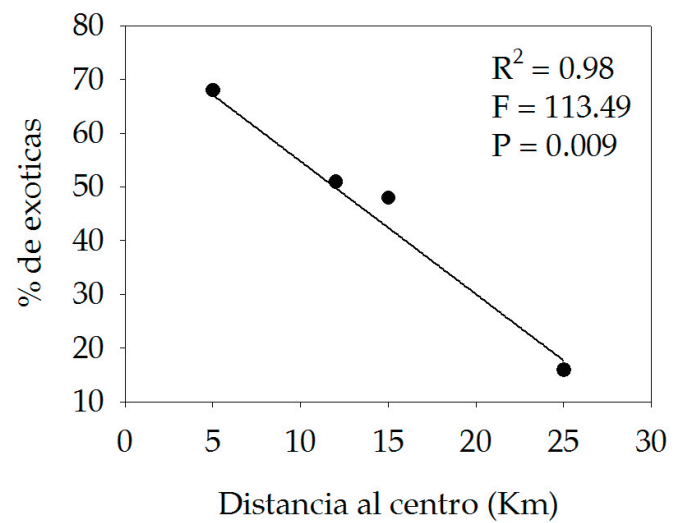

Figura 3. Relación entre la distancia al centro urbano y el porcentaje de especies exóticas. El porcentaje de cobertura de las nativas y exóticas no suman $100 \%$ porque se consideró al suelo desnudo dentro del total de cobertura del mallín.

Figure 3. Relationship between the distance of the urban center and the percentage of the exotic species.The percentage of native and exotic coverage do not add up to $100 \%$ because the bare soil is considered within the total coverage of the wetland. repens (18\%), Trifolium repens (7\%) y Potentilla anserina (3\%).

El mallín Bahía Serena es el más extenso y heterogéneo, ya que abarca 21 hectáreas (Figura 1). Se cuantificó una riqueza total de 40 especies, de las cuales el $49 \%$ fueron de origen exótico (Tabla 1, Figura 1). Sin embargo, del total de la cobertura sólo 24\% correspondió a exóticas, $40 \%$ a nativas y el resto del porcentaje $(\sim 30 \%)$ a suelo desnudo o de vegetación compacta y pisoteada por el ganado, que no pudo ser identificada. En la zona central o inundada se encontró una predominancia de Juncus bofonius (25\%), la invasora P. anserina $(8 \%)$, Plantago australis $(6 \%)$ y Trifolium repens $(5 \%)$, mientras que en la zona intermedia se encontró un bioma más arbustivo dominado por Discaria chacaye, Berberis microphylla y Escallonia virgata, y algunas especies de Juniperus sp. formando montículos de suelo donde se registró un mayor número de especies. En la zona periférica se encontró una predominancia de N. antarctica y Pinus sp.

La Laguna Fantasma (Figura 1) presentó una riqueza total de 32 especies, de las cuales $48 \%$ fueron de origen exótico (Tabla 1). En la región central, la mayor cobertura estuvo dada por la exótica invasora Potentilla anserina (47\%) y la macrófita emergente nativa Eleocharis pachycarpa (19\%). En la zona intermedia, la mayor cobertura fue de la macrófita emergente nativa Carex aematorrhyncha $(25 \%)$. En la periferia crece una variedad de especies exóticas, mayormente arbóreas plantadas por el municipio y por la junta vecinal (Fraxinus 
americana, Betula pendula, Acer pseudoplatanus y Malus domestica, entre otras).

El mallín Llao-Llao mostró una riqueza total de 25 especies, de las cuales 16\% fue de origen exótico (Tabla 1). Del total de la cobertura, más de $80 \%$ correspondió a especies nativas (Figura 2). En la región central dominan las macrófitas emergentes Isolepis ranko (17\%) y Juncus dombeyanus (17\%), mientras que en la zona intermedia dominó Carex aematorrhyncha (24\%). Por último, en la periferia del humedal se encuentran especies más arbustivas y arbóreas como Myrceugenia exucca, Luma apiculata, Nothofagus antarctica y Escallonia virgata, todas especies nativas del bosque templado húmedo.

El ordenamiento de los mallines, basado en el porcentaje de cobertura de las especies del mallín (excluyendo las especies de la periferia), analizado a través de un PCA, mostró una segregación de acuerdo con su nivel de antropización (Figura 4). Los dos primeros componentes explicaron el $82.2 \%$ de la varianza (52.5\% el PC1, 29.7\% el PC2). Doce especies fueron las que explicaron mayoritariamente la distribución de los sitios (autovalores $>0.1$ ) (Tabla 2, especies con cobertura mayor a $10 \%$ ). El eje 1 se relacionó fuertemente con la cobertura de $P$. anserina, y en menor medida con E. pachycarpa y $C$. aematorrhyncha, las tres muy abundantes en la laguna Fantasma (Tabla 2). El eje 2 se relacionó positivamente con la cobertura de C. aematorrhyncha, J. dombeyanus, I. ranko y A. stolonifera, donde se situó el mallín del Llao-Llao, y negativamente con J. bufonius, dominante en el mallín Bahía Serena y $R$.

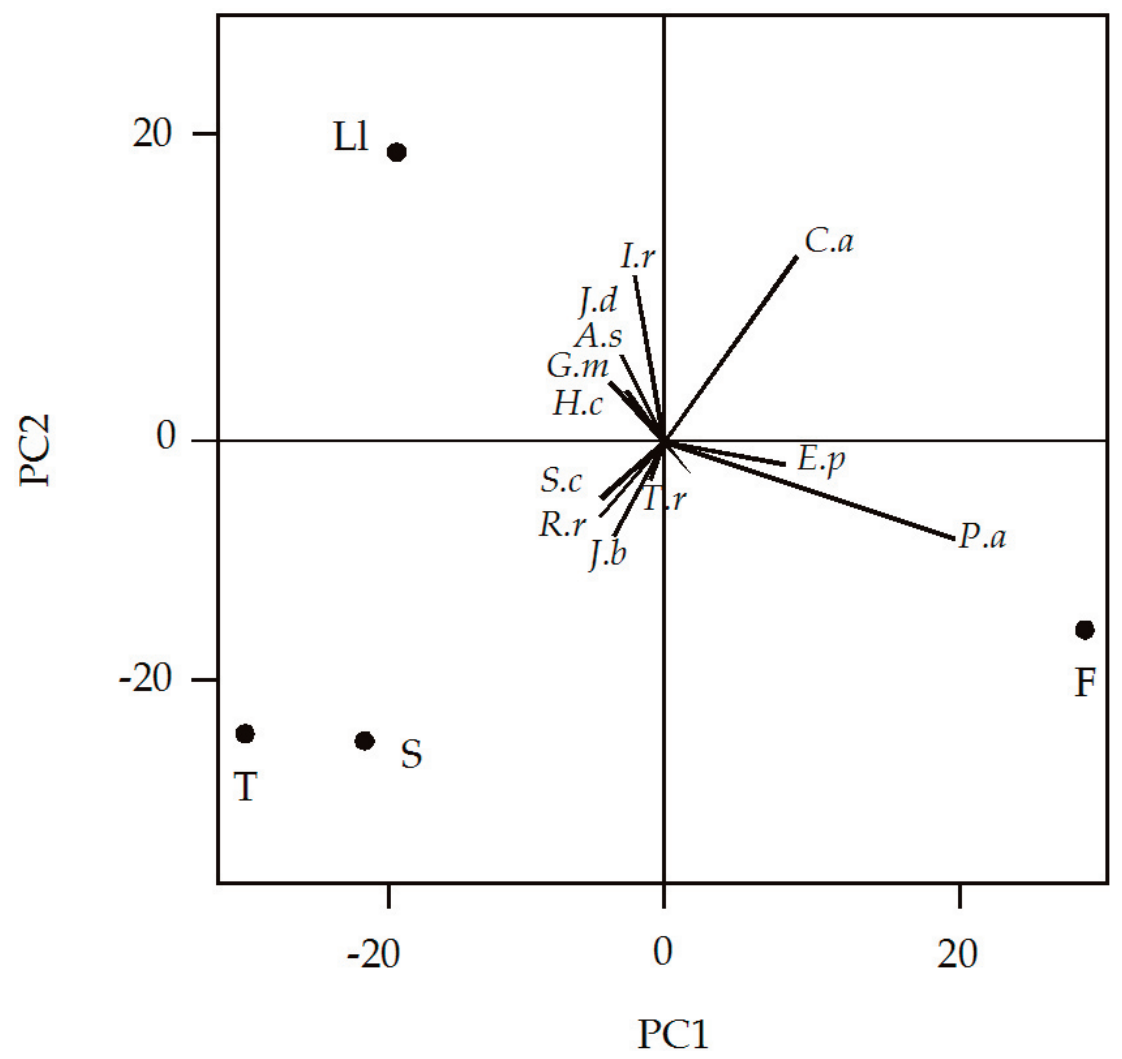

Figura 4. Ordenamiento de los cuatro mallines (T: Teleférico, S: Bahía Serena, F: Fantasma y Ll: Llao-Llao), en un análisis de componentes principales (componente 1 y 2) basado en el porcentaje de cobertura de las especies del mallín. Los vectores corresponden a las doce especies que explicaron mayoritariamente la distribución de los sitios (eigenvectors>0.1): A.s: Agrostis stolonifera, C.a: Carex aematorrhyncha, E.p: Eleocharis pachycarpa, G.m: Galium magellani cum, H.c: Hydrocotile chamaemorus, I.r: Isolepis ranko, J.d: Juncus dombeyanus, J.d: Juncus bufonius, P.a: Potentilla anserina, R.r: Ranunculus repens, S.c: Schoenoplectus californicus y T.r: Trifolium repens.

Figure 4. Ordering of the four wetlands (T: Teleférico, S: Bahía Serena, F: Fantasma y Ll: Llao-Llao), in an analysis of main components (component 1 and 2) based on the percentage of coverage of the species of the wetland. The vectors correspond to the twelve species that mostly explained the distribution of the sites (eigenvectors $>0.1$ ): A.s: Agrostis stolonifera, C.a: Carex aematorrhyncha, E.p: Eleocharis pachycarpa, G.m: Galium magellanicum, H.c: Hydrocotile chamaemorus, I.r: Isolepis ranko, J.d: Juncus dombeyanus, J.d: Juncus bufonius, P.a: Potentilla anserina, R.r: Ranunculus repens, S.c: Schoenoplectus californicus y T.r: Trifolium repens. 
repens, $S$. californicus y $T$. repens, con mayor cobertura en el mallín Teleférico (Tabla 2, Figura 4).

Considerando la riqueza específica hallada, cabe destacar que cada ambiente presentó especies exclusivas (Tabla 2). En el caso del mallín Teleférico, 11 especies fueron exclusivas, casi la totalidad de origen exótico, lo que marca un reemplazo importante de la comunidad nativa. En el mallín Bahía Serena, 16 especies fueron exclusivas de este sitio, de las cuales solo 6 fueron nativas. La laguna Fantasma sólo presentó 7 especies exclusivas, de las cuales fueron 4 nativas y 3 exóticas. Por último, el mallín Llao-Llao presentó 7 especies exclusivas, de las cuales 5 fueron nativas y 2 exóticas (Tabla 2). Además, en los tres ambientes con antropización se observó la presencia de especies exóticas invasoras como rosa mosqueta (Rosa rubiginosa) y sauce (Salix fragilis).

\section{DiscUSIÓN}

La flora de los mallines estudiados mostró un aumento del porcentaje de exóticas con la cercanía al centro de la ciudad, lo cual sustenta una de nuestras hipótesis. Del total de la riqueza hallada, un alto porcentaje fue de origen exótico (51\%), lo que indica un reemplazo de la flora nativa por la introducida y una alta susceptibilidad de los humedales a ser invadidos por especies exóticas. En el presente trabajo se demostró que la cercanía al centro de la ciudad, y por lo tanto el grado de antropización, incrementa el porcentaje de exóticas; esto señala la importancia de la urbanización en este impacto. Estos resultados coinciden con el trabajo de Speziale y Ezcurra (2011), realizado en ambientes terrestres en la misma región, pero a mayor escala. Los autores encontraron que la riqueza de especies exóticas aumentaba en la cercanía a los centros urbanos. Otro trabajo realizado en humedales urbanos en Chile mostró que aquellos con mayor número de especies nativas se hallaban más lejos de las cabeceras urbanas, lo que indicó que los principales vectores de disminución de la diversidad fueron la presencia de rutas y la densidad de casas (Rojas et al. 2015). Si bien en nuestro trabajo no encontramos una relación entre la totalidad de especies y la densidad de casas, sí observamos el aumento de las especies exóticas con la cercanía al centro de la ciudad.
Los tres ambientes que fueron considerados como antropizados presentaron distintos grados de impacto debido a los usos del suelo y a la densidad de casas (Tabla 1). Los mallines en donde se observó la presencia de ganado equino (Teleférico y Bahía Serena) presentaron un porcentaje considerable de vegetación que no pudo ser identificada, como también la presencia de suelo desnudo ( 30\% en Bahía Serena). Numerosos estudios investigaron los efectos negativos de los sitios sometidos a pastoreo donde se observó la desaparición completa de la cobertura vegetal, disminuyendo notablemente la calidad ambiental (Savory and Parsons 1980; Marty 2005; Sica et al. 2016). Estos resultados sugieren de manera indirecta (no se midió el efecto del pastoreo en este estudio de manera separada) el impacto del pastoreo sobre la cobertura vegetal, la dificultad a la hora de determinar especies y la presencia de suelo desnudo como signo de un deterioro del suelo por efecto del ganado. En este sentido, en el mallín Bahía Serena, la presencia de arbustos como E. virgata o B. microphylla, este último con espinas, generó un refugio contra el pastoreo donde se observó mayor diversidad de plantas. Esto también fue observado en el informe técnico realizado en este mallín a pedido de la Municipalidad de Bariloche (González and Alonso 2009), donde se remarca la importancia de estos "islotes de biodiversidad", lo cuales pueden ser considerados como hot spots para una posible restauración. El uso de arbustos como plantas nodrizas para especies arbóreas se sugirió como un método efectivo de reforestación en cuencas del Mediterráneo (Gómez-Aparicio et al. 2004). En los mallines pastoreados, estas nodrizas podrían estar ayudando a conservar la biodiversidad del ambiente.

La literatura menciona que los humedales son ambientes que contribuyen a la biodiversidad, ya que presentan especies endémicas de plantas (Chimner et al. 2011). En este estudio se encontró que tres de los mallines presentaron especies nativas exclusivas de cada ambiente. Ante este hallazgo, y siendo que podría tratarse de endemismos o microendemismos, sería importante considerar algún tipo de manejo o plan de conservación, ya que podrían hallarse en peligro por tratarse de especies que sólo crecen en estos ambientes. Por otro lado, estas especies también se podrían usar como indicadoras de calidad ambiental. De hecho, poseen un valor per se, ya que podrían 
ser utilizadas como indicadoras de la salud ambiental de cada humedal.

Laguna Fantasma fue la que presentó menor índice de diversidad debido al alto porcentaje de cobertura de la exótica $P$. anserina (47\%), en detrimento de la diversidad.Según Richardson (2000), las especies invasoras son aquellas que una vez introducidas en el ambiente producen descendientes reproductivos en áreas alejadas de los sitios de introducción. Considerando esta definición, P. anserina debe considerarse como una especie no sólo exótica, sino también como una especie invasora cuya presencia modifica y transforma el ambiente al conferirle al cuerpo de agua una concentración alta de nutrientes y de materia orgánica disuelta (Cuassolo et al. 2012, 2016). En consecuencia, si bien su porcentaje de cobertura en los demás mallines estudiados no supera el 10\%, el antecedente hallado en el presente trabajo en laguna Fantasma muestra el potencial invasor de esta especie, y que a largo plazo su cobertura podría aumentar.

Por otro lado, el humedal más alejado del conglomerado urbano, mallín Llao-Llao, fue el que presentó el mejor estado ambiental general y un menor porcentaje de especies exóticas (16\%), incluyendo a P. anserina, de alto potencial invasor. Su presencia se registró en el sitio de ingreso al mallín, lo cual se sugiere que las semillas pudieron ser transportadas por humanos. A pesar de esto, aquellos ambientes sin disturbio parecen ser más resistentes a las invasiones por especies exóticas, tal como se mostró en un estudio realizado en un mallín de altura (Raffaele 2004), que demostró que la riqueza y diversidad de especies nativas no se vieron afectadas por la introducción de especies exóticas, ya que las especies exóticas no se establecían con éxito en parcelas sin disturbio.

De acuerdo con la tendencia, la población de Bariloche continuará en aumento. Por lo tanto, aquellos ambientes más cercanos al centro de la ciudad o rodeados de una mayor urbanización, serán afectados por las especies invasoras. En consecuencia, se requiere una regulación mayor sobre el uso de la tierra, e incluso medidas de manejo y restauración sobre este tipo de ambientes.

Agradecimientos. Agradecemos a las instituciones que han hecho posible este estudio. Agencia Nacional de Promoción Científica y Técnica, Proyectos PICT. 2016- 4151 y PICT 2014- 1604. Un especial agradecimiento a la Dra. Cecilia Ezcurra por su aporte en la identificación de plantas. FC y VDV son investigadoras de CONICET. También agradecemos los valiosos aportes y las sugerencias de los dos revisores anónimos.

\section{REFERENCIAS}

Balseiro, E. 2014. RNU Morenito-Laguna Ezquerra. Universidad Nacional del Comahue.

Boelcke, O., and A. Vizinis. 1986. Plantas vasculares de la Argentina nativas y exóticas. FECIC. Buenos Aires.

Buono, G., M. Oesterheld, V. Nakamatsu, and J. Paruelo. 2010. Spatial and temporal variation of primary production of Patagonian wet meadows. Journal of Arid Environments 74:1257-1261. https://doi.org/10.1016/ j.jaridenv.2010.05.026.

Correa, M. 1971-1998. Flora patagónica, parte I-VIII. Colección Científica del INTA, Instituto Nacional de Tecnología Agropecuaria, Buenos Aires.

Cuassolo, F., E. Balseiro, and B. Modenutti. 2012. Alien vs. native plants in a Patagonian wetland: elemental ratios and ecosystem stoichiometric impacts. Biological invasions 14:179-189. https://doi.org/10.1007/s10530-011-9995-9.

Cuassolo, F., M. B. Navarro, E. Balseiro, and B. Modenutti. 2016. Effect of light on particulate and dissolved organic matter production of native and exotic macrophyte species in Patagonia. Hydrobiologia 766:29-42. https://doi.org/ 10.1007/s10750-015-2434-7.

Chimner, R. A., G. L. Bonvissuto, M. Cremona, J. J. Gaitan, and C. R. López. 2011. Ecohydrological conditions of wetlands along a precipitation gradient in Patagonia, Argentina. Ecologia Austral 21:329-337.

Dimitri, M. J. 1977. Pequeña flora ilustrada de los parques nacionales andino patagonicos. Buenos Aires: Servicio Nacional de Parques Nacionales. Tomo XIII. Pp. 122.

Ehrenfeld, J. G. 2000. Evaluating wetlands within an urban context. Urban Ecosystems 4:69-85. https://doi.org/ 10.1023/A:1009543920370.

Ezcurra, C., and C. Brion. 2005. Plantas del Nahuel Huapi: Catálogo de la Flora Vascular del Parque Nacional Nahuel Huapi, Argentina. Universidad Nacional del Comahue y Red Latinoamericana de Botánica San Carlos de Bariloche.

Faulkner, S. 2004. Urbanization impacts on the structure and function of forested wetlands. Urban Ecosystems 7:89-106. https://doi.org/10.1023/B:UECO.0000036269.56249.66.

Gallardo, B., M. Clavero, M. I. Sánchez, and M. Vilà. 2016. Global ecological impacts of invasive species in aquatic ecosystems. Global Change Biology 22:151-163. https://doi.org/10.1111/gcb.13004. 
Gómez-Aparicio, L., R. Zamora, J. M. Gómez, J. A. Hódar, J. Castro, and E. Baraza. 2004. Applying plant facilitation to forest restoration: a meta'analysis of the use of shrubs as nurse plants. Ecological Applications 14:1128-1138. https: //doi.org/10.1890/03-5084.

González, M., and M. Alonso. 2009. Caracterización de la situación ambiental del humedal Bahía Serena, mallín del km 12, San Carlos de Bariloche. Universidad Nacional del Comahue.

Guevara, S. R., M. A. Arribére, D. Bubach, P. Vigliano, A. Rizzo, M. Alonso, and R. Sánchez. 2005. Silver contamination on abiotic and biotic compartments of Nahuel Huapi National Park lakes, Patagonia, Argentina. Science of The Total Environment 336:119-134. https://doi.org/10.1016/j.scitotenv.2004.05.020.

Harguinteguy, C. A., R. Schreiber, and M. L. Pignata. 2013. Myriophyllum aquaticum as a biomonitor of water heavy metal input related to agricultural activities in the Xanaes River (Córdoba, Argentina). Ecological Indicators 27:8-16. https://doi.org/10.1016/j.ecolind.2012.11.018.

Iriondo, M., J. Orellana, and J. Neiff. 1974. Sobre el concepto de mallín cordillerano. Revista de la Asociación de Ciencias Naturales del Litoral (Argentina) 5:45-52. https://doi.org/10.14409/natura.v1i5.3275.

Jara, F. G., C. A. Úbeda, and M. G. Perotti. 2013. Predatory insects in lentic freshwater habitats from northwest Patagonia: richness and phenology. Journal of natural history 47:2749-2768. https://doi.org/10.1080/00222933.2013.791932.

Lockwood, J. L., P. Cassey, and T. M. Blackburn. 2009. The more you introduce the more you get: the role of colonization pressure and propagule pressure in invasion ecology. Diversity and distributions 15:904-910. https://doi.org/10.1111/ j.1472-4642.2009.00594.x.

Marty, J. T. 2005. Effects of cattle grazing on diversity in ephemeral wetlands. Conservation Biology 19:1626-1632. https: //doi.org/10.1111/j.1523-1739.2005.00198.x.

Matteucci, S. D., and A. Colma. 1982. Metodología para el estudio de la vegetación. The General Secretariat of the Organization of American States. USA. Pp. 163.

Mazzarino, M., M. Bertiller, T. Schlichter, and M. Gobbi. 1998. Nutrient cycling in Patagonian ecosystems. Ecologia Austral 8:167-181.

Paruelo, J. M., A. Beltrán, E. Jobbágy, O. E. Sala, and R. A. Golluscio. 1998. The climate of Patagonia: general patterns and controls on biotic. Ecologia Austral 8:85-101.

Perotti, M. G., M. C. Diéguez, and F. G. Jara. 2005. Estado del conocimiento de humedales del norte patagónico (Argentina): aspectos relevantes e importancia para la conservación de la biodiversidad regional. Revista chilena de historia natural 78:723-737. https://doi.org/10.4067/S0716-078X2005000400011.

Raffaele, E. 1999. Mallines: aspectos generales y problemas particulares. Topicos sobre humedales subtropicales y templados de Sudamerica. Oficina Regional de Ciencia y Tecnologia de la UNESCO para America Latina y el Caribe. ORCYT, Montevideo, Uruguay. Google Scholar:27-33.

Raffaele, E. 2004. Susceptibility of a Patagonian mallín flooded meadow to invasion by exotic species. Biological invasions 6:473-481. https://doi.org/10.1023/B:BINV.0000041560.33770.97.

Ramírez, C., C. San Martín, and H. Rubilar. 2002. Una propuesta para la clasificación de los humedales chilenos. Revista Geográfica de Valparaíso 33:265-273.

Ramsar. 2006. Manual de la Convención de Ramsar: Guía a la Convención sobre los Humedales (Ramsar, Irán, 1971). Secretaría de la Convención de Ramsar, Gland, Suiza.

Richardson, D. M., P. Pyšek, M. Rejmánek, M. G. Barbour, F. D. Panetta, and C. J. West. 2000. Naturalization and invasion of alien plants: concepts and definitions. Diversity and distributions 6:93-107. https://doi.org/10.1046/j.14724642.2000.00083.x.

Rojas, C., E. Sepúlveda-Zúñiga, O. Barbosa, O. Rojas, and C. Martínez. 2015. Patrones de urbanización en la biodiversidad de humedales urbanos en Concepción metropolitano. Revista de Geografía Norte Grande 61:181-204. https://doi.org/ $10.4067 / S 0718-34022015000200010$

Sala, O. E. 2000. Global Biodiversity Scenarios for the Year 2100. Science 287:1770-1774. https://doi.org/10.1126/ science.287.5459.1770.

Satti, P., M. J. Mazzarino, M. Gobbi, F. Funes, L. Roselli, and H. Fernández. 2003. Soil N dynamics in relation to leaf litter quality and soil fertility in north-western Patagonian forests. Journal of Ecology 91:173-181. https://doi.org/ 10.1046/j.1365-2745.2003.00756.x.

Savory, A., and S. D. Parsons. 1980. The Savory grazing method. Rangelands 2:234-237.

Sica, Y., R. Quintana, V. Radeloff, and G. Gavier-Pizarro. 2016. Wetland loss due to land use change in the Lower Paraná River Delta, Argentina. Science of The Total Environment 568:967-978. https://doi.org/10.1016/ j.scitotenv.2016.04.200.

Simberloff, D., J.-L. Martin, P. Genovesi, V. Maris, D. A. Wardle, J. Aronson, F. Courchamp, B. Galil, E. García-Berthou, and M. Pascal. 2013. Impacts of biological invasions: what's what and the way forward. Trends in Ecology and Evolution 28:58-66. https://doi.org/10.1016/j.tree.2012.07.013.

Speziale, K., and C. Ezcurra. 2011. Patterns of alien plant invasions in northwestern Patagonia, Argentina. Journal of Arid Environments 75:890-897. https://doi.org/10.1016/j.jaridenv.2011.04.014.

Vitousek, P. M. 1990. Biological invasions and ecosystem processes: towards an integration of population biology and ecosystem studies. Pp. 183-191 in Ecosystem Management. Springer. https://doi.org/10.1007/978-1-4612-4018-1_17.

Zedler, J. B., and S. Kercher. 2004. Causes and consequences of invasive plants in wetlands: opportunities, opportunists, and outcomes. Critical Reviews in Plant Sciences 23:431-452. https://doi.org/10.1080/07352680490514673. 